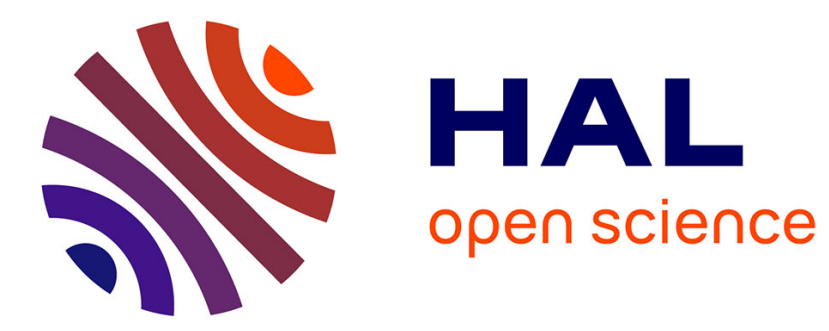

\title{
On the Input-Output Distinguishability of Single Output Continuous Linear Time-Invariant Systems
}

Koffi Mawussé Djidula Motchon, Komi Midzodzi Pekpe, Jean Philippe Cassar, Stephan De Bièvre

\section{- To cite this version:}

Koffi Mawussé Djidula Motchon, Komi Midzodzi Pekpe, Jean Philippe Cassar, Stephan De Bièvre. On the Input-Output Distinguishability of Single Output Continuous Linear Time-Invariant Systems. IEEE Transactions on Automatic Control, 2015, 61 (99), pp.1906-1911. 10.1109/TAC.2015.2481788 . hal-01280900

\section{HAL Id: hal-01280900 https://hal.science/hal-01280900}

Submitted on 1 Mar 2016

HAL is a multi-disciplinary open access archive for the deposit and dissemination of scientific research documents, whether they are published or not. The documents may come from teaching and research institutions in France or abroad, or from public or private research centers.
L'archive ouverte pluridisciplinaire HAL, est destinée au dépôt et à la diffusion de documents scientifiques de niveau recherche, publiés ou non, émanant des établissements d'enseignement et de recherche français ou étrangers, des laboratoires publics ou privés. 


\section{On the Input-Output Distinguishability of Single Output Continuous Linear Time-Invariant Systems}

Koffi M. D. Motchon, Komi M. Pekpe, Jean-Philippe Cassar, and Stephan De Bièvre

\begin{abstract}
This technical note addresses the distinguishability problem for continuous linear time-invariant systems using their input and output data. The proposed approach is based on the characterization of the class of initial state vectors and control input signals that make the outputs of different continuous linear time-invariant systems indistinguishable. This class is defined here as the "indistinguishability zone". From this characterization, a simple necessary and sufficient condition for the distinguishability of single-input, single-output systems is established. Furthermore, it is shown that multiple-input, single-output systems are never distinguishable.
\end{abstract}

Index Terms-Linear time-invariant system, distinguishability, Markov parameters, Switched systems.

\section{INTRODUCTION}

The property of distinguishability of two systems refers to their capacity to generate different output signals for a given input signal. This property was firstly introduced in the 1970s by Grewal et al. [1], [2], who have shown its importance for the identifiability of dynamical systems. Distinguishability is also important when studying the observability of switched systems as underlined recently in [3], [4], [5]. It characterizes in this context the ability to determine the active mode of the system from the input and output data.

Several notions of distinguishability may be encountered in the literature, depending on the field of application. In this technical note, we call "strict distinguishability" the notion of distinguishability proposed in [4]. Two linear continuous time-invariant (LTI) systems are strictly distinguishable if for any initial state vectors and control inputs of the systems (not both zero), their corresponding outputs are different. An adaptation of this notion of distinguishability for discrete LTI dynamical systems is proposed in [6].

Studies of strict distinguishability deal with the determination of necessary and (or) sufficient conditions that allow to test whether or not two different systems are strictly distinguishable. These conditions are often "rank conditions" (see for example [4], [6], [7]) on an appropriate matrix that depends on the matrices used to model the systems in a state-space representation. However, to the best of our knowledge, there is currently no research on the determination of the class of initial state vectors and input signals that generate identical output signals for dynamical systems which are not strictly distinguishable, a problem we will attack here.

Specifically, this paper is concerned with the construction of the set of initial state vectors and control inputs that lead to indistinguishable outputs for two different continuous LTI systems. We will introduce the notion of "indistinguishability zone" to refer to this class.

The paper is organized as follows. Section II is devoted to the formulation of the problem. It also contains the preliminary results necessary to obtain the characterization of the indistinguishability zone given in Section III. This characterization is used in Section IV to establish simple conditions for strict distinguishability of singleinput, single-output (SISO) and multiple-input, single-output (MISO)

K. Motchon, K. Pekpe and J-P. Cassar are with CRIStAL UMR CNRS 9189, Université Lille 1, 59650 Villeneuve d'Ascq Cedex France (e-mail: kmd.motchon@ed.univ-lille1.fr, midzodzi.pekpe@univ-lille1.fr and jean-philippe.cassar@univ-lille1.fr).

S. De Bièvre is with Lab. Paul Painlevé UMR CNRS 8524 and EPI MEPHYSTO INRIA Lille Nord-Europe, Université Lille 1, 59650 Villeneuve d'Ascq Cedex France (e-mail: Stephan.De-Bievre@math.univ-lille1.fr). systems. Finally, some remarks and conclusions are highlighted in Section V.

For more information on the state of the art and examples illustrating the theory, the interested reader can inspect [8].

Throughout the remainder of this paper, we adopt the following notation, for all $m_{1}, m_{2} \in \mathbb{N}^{\star}$ :

- $\mathbb{R}_{+}=[0 ;+\infty[$ : set of positive real numbers.

- $\mathbb{R}^{m_{1}}$ : Euclidean $m_{1}$-space ; $0_{m_{1}}$ : zero vector of $\mathbb{R}^{m_{1}}$.

- $I_{m_{1}}$ : identity matrix of $\mathbb{R}^{m_{1} \times m_{1}}$.

- $\mathbb{R}^{m_{1} \times m_{2}}$ : space of matrices with $m_{1}$ rows and $m_{2}$ columns.

- $0_{m_{1} \times m_{2}}$ : zero matrix of $\mathbb{R}^{m_{1} \times m_{2}}$.

- $\mathbb{R}^{m_{1} \times(\bullet)}$ : space of matrices with $m_{1}$ rows.

- $\operatorname{deg}(\cdot)$ : degree function.

- $\mathscr{H}_{\gamma}=\{s \in \mathbb{C}: \Re(s)>\gamma\}$ : half-plane to the right of the vertical line $\Re(s)=\gamma$ where $\Re(s)$ denotes the real part of $s$.

- $\mathscr{C}_{\mathscr{P}}^{\text {ord }}\left(\mathbb{R}_{+}, \mathbb{R}^{m_{1}}\right)$ : set of functions from $\mathbb{R}_{+}$to $\mathbb{R}^{m_{1}}$ that are continuous and piecewise differentiable on $\mathbb{R}_{+}$and that have an exponential order at infinity (see [9]).

- *: convolution product.

\section{STATEMENT OF THE PROBLEM AND PRELIMINARIES}

\section{A. Statement of the problem}

Consider two different single-output linear time-invariant systems $S_{1}$ and $S_{2}$ of the form

$$
S_{i}\left\{\begin{array}{l}
\dot{x}_{i}(t)=A_{i} x_{i}(t)+B_{i} u(t), \\
y_{i}(t)=C_{i} x_{i}(t), \\
x_{i}(0)=x_{i}^{o},
\end{array}\right.
$$

where $x_{i}(t) \in \mathbb{R}^{n}$ and $y_{i}(t) \in \mathbb{R}$ are respectively the state vector and the output vector of $S_{i}$ and $u(t) \in \mathbb{R}^{l}$ is the input vector conjointly applied to $S_{1}$ and $S_{2} ; A_{i}, B_{i}$ and $C_{i}$ are constant matrices with appropriate dimensions.

In the rest of this note, we denote by $y_{i}\left(\cdot, x_{i}^{o}, u\right)$ the output signal $y_{i}$ of $S_{i}$ corresponding to the initial state vector $x_{i}^{o}$ and the control input $u$. The indistinguishability zone of $S_{1}$ and $S_{2}$ is defined as follows.

Definition 1. The indistinguishability zone, $\mathscr{Z}_{\text {ind }}\left(S_{1}, S_{2}\right)$ of the systems $S_{1}$ and $S_{2}$ is the subset of $\mathbb{R}^{n} \times \mathbb{R}^{n} \times \mathscr{U}$ given by

$$
\mathscr{Z}_{\text {ind }}\left(S_{1}, S_{2}\right)=\left\{\left(x_{1}^{o}, x_{2}^{o}, u\right): y_{1}\left(\cdot, x_{1}^{o}, u\right) \equiv y_{2}\left(\cdot, x_{2}^{o}, u\right)\right\}
$$

where $\mathscr{U}$ denotes the set of admissible inputs of the two systems and the relation $y_{1}\left(\cdot, x_{1}^{o}, u\right) \equiv y_{2}\left(\cdot, x_{2}^{o}, u\right)$ means that the signals $y_{1}$ and $y_{2}$ are identical on $\mathbb{R}_{+}$.

Example 2. Consider two harmonic systems $S_{1}$ and $S_{2}$ which have the following state-space representation:

$S_{i}\left\{\begin{array}{l}{\left[\begin{array}{l}\dot{q}_{i}(t) \\ \ddot{q}_{i}(t)\end{array}\right]=\left[\begin{array}{cc}0_{\tilde{n} \times \tilde{n}} & I_{\tilde{n}} \\ -M_{i}^{-1} K_{i} & 0_{\tilde{n} \times \tilde{n}}\end{array}\right]\left[\begin{array}{l}q_{i}(t) \\ \dot{q}_{i}(t)\end{array}\right]+\left[\begin{array}{c}0_{\tilde{n} \times \tilde{n}} \\ M_{i}^{-1}\end{array}\right] u(t),} \\ y_{i}(t)=q_{i}(t),\end{array}\right.$

where $M_{i} \in \mathbb{R}^{\tilde{n} \times \tilde{n}}$ and $K_{i} \in \mathbb{R}^{\tilde{n} \times \tilde{n}}$ are real, symmetric positivedefinite matrices. One may determine the indistinguishability zone of $S_{1}$ and $S_{2}$ as follows. Let $\left(x_{1}^{o}, x_{2}^{o}, u\right) \in \mathscr{Z}_{\text {ind }}\left(S_{1}, S_{2}\right)$. Then $q_{1} \equiv q_{2}=: q$ and this implies that $\ddot{q}_{1} \equiv \ddot{q}_{2} \equiv \ddot{q}$. Consequently, when $M_{2}-M_{1}$ is invertible, we deduce from the following expression

$$
\ddot{q}_{i}(t)=-M_{i}^{-1} K_{i} q_{i}(t)+M_{i}^{-1} u(t), \quad i=1,2,
$$

which derives from (2) that $u(t)$ and $q(t)$ satisfy

$$
u(t)=G q(t)
$$


where $G=\left(M_{2}^{-1}-M_{1}^{-1}\right)^{-1}\left(M_{2}^{-1} K_{2}-M_{1}^{-1} K_{1}\right)$. By substituting $u(t)=G q(t)$ into (3) for $i=1$, one obtains that $q$ is the solution of the second order differential equation $\ddot{q}=-H q$ with $H:=M_{1}^{-1} K_{1}-M_{1}^{-1} G=M_{2}^{-1} K_{2}-M_{2}^{-1} G$. Let us suppose $\sqrt{H}$ exists and is invertible. Then one obtains

$$
q(t)=\cos (t \sqrt{H}) q^{o}+(\sqrt{H})^{-1} \sin (t \sqrt{H}) \dot{q}^{o}
$$

with $q^{o}:=q(0)=q_{1}(0)=q_{2}(0)$ and $\dot{q}^{o}:=\dot{q}(0)=\dot{q}_{1}(0)=\dot{q}_{2}(0)$. Therefore, the expression (4) of $u$ becomes:

$$
u(t)=G \cos (t \sqrt{H}) q^{o}+G(\sqrt{H})^{-1} \sin (t \sqrt{H}) \dot{q}^{o} .
$$

We conclude that if $\left(x_{1}^{o}, x_{2}^{o}, u\right) \in \mathscr{Z}_{\text {ind }}\left(S_{1}, S_{2}\right)$ then $x_{1}^{o}=x_{2}^{o}$ (i.e. $q_{1}(0)=q_{2}(0)=: q^{o}$ and $\left.\dot{q}_{1}(0)=\dot{q}_{2}(0)=: \dot{q}^{o}\right)$ and $u$ is given explicitly by (5).

Conversely, suppose $x_{1}^{o}=x_{2}^{o}$ and that $u$ is given by (5). Then, $u(t)=G q(t)$ with $q$ solution of $\ddot{q}=-H q$. Thus, from the formula of $H$ and (3), one obtains $\ddot{q}_{1} \equiv \ddot{q} \equiv \ddot{q}_{2}$. As $x_{1}^{o}=x_{2}^{o}$, one has $q_{1}(0)=$ $q_{2}(0)$ and $\dot{q}_{1}(0)=\dot{q}_{2}(0)$. Consequently, the relation $\ddot{q}_{1} \equiv \ddot{q}_{2}$ implies that $q_{1} \equiv q_{2}$. Hence $y_{1}\left(\cdot, x_{1}^{o}, u\right)$ and $y_{2}\left(\cdot, x_{2}^{o}, u\right)$ are identical on $\mathbb{R}_{+}$.

Remark 3. The zero triplet of $\mathbb{R}^{n} \times \mathbb{R}^{n} \times \mathscr{U},\left(0_{n}, 0_{n}, 0_{\mathscr{U}}\right)$ with $0_{\mathscr{U}}$ the zero function of $\mathscr{U}$, always belongs to $\mathscr{Z}_{\text {ind }}\left(S_{1}, S_{2}\right)$.

In the sequel of the paper, we will focus on the determination of the triplets $\left(x_{1}^{o}, x_{2}^{o}, u\right)$ that belong to the indistinguishability zone $\mathscr{Z}_{\text {ind }}\left(S_{1}, S_{2}\right)$ of $S_{1}$ and $S_{2}$ and deduce the existence conditions of non-zero triplets. Throughout the remainder of this technical note, we assume that

Assumption 4. $\mathscr{U}=\mathscr{C}_{\mathscr{P}}^{\text {ord }}\left(\mathbb{R}_{+}, \mathbb{R}^{l}\right)$.

This assumption ensures that the control inputs $u$ considered in the paper are Laplace transformable [9], [10].

\section{B. Preliminaries}

The aim of this section is to establish some fundamental results which are necessary to characterize, in the next section, the indistinguishability zone of $S_{1}$ and $S_{2}$. Lemma 5 is the main tool for our subsequent analysis. It gives a first characterization of the indistinguishability zone. For simplicity in the presentation, we will consider the augmented (or extended) system $S$ associate to $S_{1}$ and $S_{2}$ defined by:

$$
S\left\{\begin{array}{l}
\dot{x}(t)=A x(t)+B u(t) \\
y(t)=C x(t) \\
x(0)=x_{o}
\end{array}\right.
$$

where the matrices $A, B$ and $C$ are defined as follows:

$$
A=\left[\begin{array}{ll}
A_{1} & 0_{n} \\
0_{n} & A_{2}
\end{array}\right] \quad ; \quad B=\left[\begin{array}{l}
B_{1} \\
B_{2}
\end{array}\right] \quad ; \quad C=\left[\begin{array}{ll}
C_{1} & -C_{2}
\end{array}\right]
$$

and

$$
x_{o}=\left[\begin{array}{ll}
\left(x_{1}^{o}\right)^{T} & \left(x_{2}^{o}\right)^{T}
\end{array}\right]^{T}=\left[\begin{array}{l}
x_{1}^{o} \\
x_{2}^{o}
\end{array}\right]
$$

is the initial state vector of $S$. As for the systems $S_{1}$ and $S_{2}$, we denote by $y\left(\cdot, x_{o}, u\right)$ the output signal of $S$ corresponding to the initial state vector $x_{o}$ and the control input $u$. Thus the output $y\left(\cdot, x_{o}, u\right)$ of $S$ is the difference $y_{1}\left(\cdot, x_{1}^{o}, u\right)-y_{2}\left(\cdot, x_{2}^{o}, u\right)$ of the $S_{1}$ and $S_{2}$ output signals. Consequently, $\left(x_{1}^{o}, x_{2}^{o}, u\right) \in \mathscr{Z}_{\text {ind }}\left(S_{1}, S_{2}\right)$ if and only if

$$
y\left(t, x_{o}, u\right)=0 \quad \forall t \in \mathbb{R}_{+} .
$$

One can easily show that $y\left(\cdot, x_{o}, u\right)$ has exponential order $\theta_{y}$, for any $\theta_{y} \geq \boldsymbol{\lambda}^{\star}+\theta_{u}$ where $\boldsymbol{\lambda}^{\star}=\max _{\lambda \in \sigma(A)} \Re(\lambda)$ with $\sigma(A)$ the spectrum of $A$ and where $\theta_{u}$ denotes any exponential order of $u$. Hence it follows from Lerch's Theorem [11] that the condition in (7) holds if and only if there exists a real number $\theta>\boldsymbol{\lambda}^{\star}+\theta_{u}$ such that

$$
Y\left(s, x_{o}, u\right)=0 \quad \forall s \in \mathscr{H}_{\theta}
$$

where $Y\left(\cdot, x_{o}, u\right)$ denotes the Laplace transform of the output $y\left(\cdot, x_{o}, u\right)$. It is straightforward to verify that $\forall s \in \mathscr{H}_{\boldsymbol{\lambda}^{\star}+\theta_{u}}$,

$$
Y\left(s, x_{o}, u\right)=\frac{\mathrm{F}(s, C, A, B) U(s)+\mathrm{F}\left(s, C, A, x_{o}\right)}{\operatorname{det}\left(s I_{2 n}-A\right)}
$$

where for every $s \in \mathscr{H}_{\lambda^{\star}}$, the operator $\mathrm{F}(s, C, A, \cdot)$ is defined on $\mathbb{R}^{2 n \times(\bullet)}$ by

$$
M \in \mathbb{R}^{2 n \times(\bullet)}, \quad \mathrm{F}(s, C, A, M)=C \operatorname{adj}\left(s I_{2 n}-A\right) M
$$

with $\operatorname{adj}\left(s I_{2 n}-A\right)=\operatorname{det}\left(s I_{2 n}-A\right)\left(s I_{2 n}-A\right)^{-1}$.

Lemma 5 introduces a condition under which the triplet $\left(x_{1}^{o}, x_{2}^{o}, u\right)$ belongs to the indistinguishability zone of $S_{1}$ and $S_{2}$.

Lemma 5. Let $\left(x_{1}^{o}, x_{2}^{o}, u\right) \in \mathbb{R}^{n} \times \mathbb{R}^{n} \times \mathscr{U}$. Then the following statements are equivalent:

(i) $\left(x_{1}^{o}, x_{2}, u\right) \in \mathscr{Z}_{\text {ind }}\left(S_{1}, S_{2}\right)$.

(ii) There exists a real number $\theta$ such that $\theta>\boldsymbol{\lambda}^{\star}+\theta_{u}$ and

$$
\mathrm{F}(s, C, A, B) U(s)+\mathrm{F}\left(s, C, A, x_{o}\right)=0
$$

for every $s \in \mathscr{H}_{\theta}$.

This result directly derives from (9) and from the equivalence between (7) and (8). To characterize the indistinguishability zone of $S_{1}$ and $S_{2}$ using Lemma 5 , the polynomial forms of $\mathrm{F}(\cdot, C, A, B)$ and $\mathrm{F}\left(\cdot, C, A, x_{o}\right)$ given by (13) are needed. This relation can be established using the following expression of $\operatorname{adj}\left(s I_{2 n}-A\right)$ [12], [13]:

$$
\operatorname{adj}\left(s I_{2 n}-A\right)=\mathrm{Q}_{2 n-1} s^{2 n-1}+\cdots+\mathrm{Q}_{1} s+\mathrm{Q}_{0}
$$

where the matrices $\mathrm{Q}_{k} \in \mathbb{R}^{2 n \times 2 n}, k=0, \ldots, 2 n-1$ are defined by

$$
\left\{\begin{array}{l}
\mathrm{Q}_{2 n-1}=I_{2 n} \\
\mathrm{Q}_{2 n-1-r}=\alpha_{2 n-r} I_{2 n}+Q_{2 n-r} A, \quad r=1,2, \ldots, 2 n-1
\end{array}\right.
$$

and the $\alpha_{k}, k=0,1, \ldots, 2 n-1$ denote the coefficients of the characteristic polynomial of $A$ i.e.

$$
\operatorname{det}\left(s I_{2 n}-A\right)=s^{2 n}+\alpha_{2 n-1} s^{2 n-1}+\cdots+\alpha_{1} s+\alpha_{0} .
$$

For every $s \in \mathscr{H}_{\boldsymbol{\lambda}^{\star}}, \mathrm{F}(s, C, A, M)$ can be written in the polynomial form:

$$
\mathrm{F}(s, C, A, M)=\sum_{r=0}^{2 n-1} \mathrm{D}_{2 n-1-r}(M) s^{2 n-1-r} .
$$

It follows from (10) and (12) that, for every $r=0,1, \ldots, 2 n-1$, the matrices $\mathrm{D}_{2 n-1-r}(M)=C \mathrm{Q}_{2 n-1-r} M$ can be expanded as follows:

$$
\mathrm{D}_{2 n-1-r}(M)=\sum_{k=0}^{r} \alpha_{2 n-r+k} C A^{k} M
$$

with $\alpha_{2 n}=1$.

Lemma 6 below gives the crucial properties of the matrix $\mathrm{F}(s, C, A, M)$ that are needed in the next sections.

Lemma 6. Let $M \in \mathbb{R}^{n \times q}$. Then for every $r_{0} \in\{0,1, \ldots, 2 n-1\}$, the following statements are equivalent:

(i) $\forall r=0,1, \ldots, r_{0}, \quad \mathrm{D}_{2 n-1-r}(M)=0_{1 \times q}$.

(ii) $\forall r=0,1, \ldots, r_{0}, \quad C A^{r} M=0_{1 \times q}$.

Proof. cf. Appendix A 
The matrix $C A^{k} B=C_{1} A_{1}^{k} B_{1}-C_{2} A_{2}^{k} B_{2}$ that appears in the formula of $\mathrm{F}(s, C, A, B)$ corresponds to the $k^{\text {th }}$ Markov parameter of $S$. Relatively to the Markov parameters of $S$, consider the index $k_{0}$ defined by

$$
k_{0}=\left\{\begin{array}{ll}
\min \left(\mathscr{E}_{\mathbb{N}}\right) & \text { if } \\
2 n & \text { if not, }
\end{array} \mathscr{E}_{\mathbb{N}} \neq \emptyset\right.
$$

where $\mathscr{E}_{\mathbb{N}}=\left\{k \in \mathbb{N}: C A^{k} B \neq 0_{1 \times l}\right\}$.

Example 7. Consider the two harmonic systems of Example 2 and suppose $\tilde{n}=1$ (i.e. $n=2$ ). One has $k_{0}=1$ if $M_{1} \neq M_{2} ; k_{0}=3$ if $M_{1}=M_{2}$ and $K_{1} \neq K_{2}$; and $k_{0}=4$ if $M_{1}=M_{2}$ and $K_{1}=K_{2}$.

The index $k_{0}$ provides information on the similarity between $S_{1}$ and $S_{2}$ in terms of their Markov parameters. Using the well known Cayley-Hamilton theorem, it is easy to verify that $0 \leq k_{0} \leq 2 n$. Therefore, the following three cases will be discussed in the next sections:

- case $1: k_{0}=2 n$.

- case $2: k_{0}=2 n-1$.

- case 3: $0 \leq k_{0}<2 n-1$.

The first case corresponds to the situation where $S_{1}$ and $S_{2}$ have the same Markov parameters. In the second case, only the $(2 n-1)^{\text {th }}$ Markov parameter of $S_{1}$ and $S_{2}$ are different. The last case corresponds to the case where there exists at least one couple of Markov parameters of the same order $k<2 n-1$ that are different.

\section{CharaCterization OF THE INDistinguishabiLity ZONE}

This section aims to characterize the indistinguishability zone of $S_{1}$ and $S_{2}$. The case where $k_{0}=2 n$ and the case where $k_{0}=2 n-1$ are considered first in Theorem 9. The more complex, and more generic, situation where $k_{0}<2 n-1$ is discussed in Theorem 16. In the sequel of the paper, we adopt the following notation:

\section{Notation 8.}

- $B=\left[\begin{array}{llll}\mathbf{B}^{1} & \mathbf{B}^{2} & \ldots & \mathbf{B}^{l}\end{array}\right]$ where $\mathbf{B}^{j}$ denotes the $j^{\text {th }}$ column of $B$.

- $\mathcal{O}^{[k]}=\left[\begin{array}{llll}C^{T} & (C A)^{T} & \ldots & \left(C A^{k}\right)^{T}\end{array}\right]^{T}$ denotes the observability matrix at order $k \in \mathbb{N}$ of $S$.

- $\mathcal{O}:=\mathcal{O}^{[2 n-1]}$ denotes the observability matrix of $S$.

A. Characterization of the indistinguishability zone: the cases $k_{0}=2 n$ and $k_{0}=2 n-1$.

When $k_{0}=2 n$ or $k_{0}=2 n-1$, the indistinguishability zone of $S_{1}$ and $S_{2}$ can be characterized as follows:

\section{Theorem 9.}

(i) If $k_{0}=2 n$ then $\left(x_{1}^{o}, x_{2}^{o}, u\right) \in \mathscr{Z}_{\text {ind }}\left(S_{1}, S_{2}\right)$ if and only if

$$
\left[\left(x_{1}^{o}\right)^{T} \quad\left(x_{2}^{o}\right)^{T}\right]^{T} \in \operatorname{Ker}(\mathcal{O}) .
$$

(ii) If $k_{0}=2 n-1$ then $\left(x_{1}^{o}, x_{2}^{o}, u\right) \in \mathscr{Z}_{\text {ind }}\left(S_{1}, S_{2}\right)$ if and only if $x_{1}^{o}$ and $x_{2}^{o}$ satisfy (16) and

$$
u \in\left\{v \in \mathscr{U}: \forall t \in \mathbb{R}_{+}, \quad v(t) \in \operatorname{Ker}\left(C A^{2 n-1} B\right)\right\} .
$$

Proof.

(i) Suppose $k_{0}=2 n$. Then from the definition (15) of $k_{0}$, $C A^{r} B=0_{1 \times l}$ for every $r=0,1, \ldots, 2 n-1$. Consequently from Lemma 6 , one has $\mathrm{D}_{2 n-1-r}(B)=0_{1 \times q}$ for every $r=$ $0,1, \ldots, 2 n-1$. Therefore $\mathrm{F}(\cdot, C, A, B)$ vanishes identically and from Lemma 5 , one has $\left(x_{1}^{o}, x_{2}^{o}, u\right) \in \mathscr{Z}_{\text {ind }}\left(S_{1}, S_{2}, \mathbb{R}_{+}\right)$ if and only if the polynomial matrix function $\mathrm{F}\left(\cdot, C, A, x_{o}\right)$ vanishes identically. Moreover as one can deduce from Lemma 6 and (13) - (14) that $\mathrm{F}\left(\cdot, C, A, x_{o}\right)$ vanishes identically if and only if $C A^{r} x_{0}=0$ for every $r=0,1, \ldots, 2 n-1$. Hence (16) holds.

(ii) Suppose $k_{0}=2 n-1$. To begin with the proof, notice that as $C A^{r} B=0_{1 \times l}$ for every $r=0,1, \ldots, 2 n-2$ then it follows from Lemma 6 that $\mathrm{D}_{2 n-1-r}(B)=0_{1 \times l}$ for every $r=0,1, \ldots, 2 n-2$. Consequently,

$$
\mathrm{F}(s, C, A, B)=\mathrm{D}_{0}(B)=C A^{2 n-1} B \quad \forall s \in \mathscr{H}_{\boldsymbol{\lambda}^{\star}} .
$$

Now let $\left(x_{1}^{o}, x_{2}^{o}, u\right) \in \mathscr{Z}_{i n d}\left(S_{1}, S_{2}\right)$. Its follows from (18) and from Lemma 5 that there exists a real $\theta>\boldsymbol{\lambda}^{\star}+\theta_{u}$ such that

$$
C A^{2 n-1} B U(s)=-\mathrm{F}\left(s, C, A, x_{o}\right) \quad \forall p \in \mathscr{H}_{\theta} .
$$

Since $u$ is continuous, it follows from the initial value theorem [9] that $C A^{2 n-1} B u(0)=-\lim _{s \rightarrow+\infty} s \mathrm{~F}\left(s, C, A, x_{o}\right)$. Since $\mathrm{F}\left(\cdot, C, A, x_{o}\right)$ is a polynomial, this can be true only if $\mathrm{F}\left(\cdot, C, A, x_{o}\right)$ vanishes identically. Thus, $\mathrm{D}_{2 n-1-r}\left(x_{o}\right)=0$ for every $r=0,1, \ldots, 2 n-1$. Consequently we conclude from Lemma 6 that (16) holds. Finally, (19) becomes

$$
C A^{2 n-1} B U(s)=0, \quad \forall s \in \mathscr{H}_{\theta} \subset \mathscr{H}_{\boldsymbol{\lambda}^{\star}+\theta_{u}} \subset \mathscr{H}_{\theta_{u}},
$$

and we conclude from Lerch's Theorem that $C A^{2 n-1} B u(t)=$ 0 for every $t \geq 0$. This shows that $u$ satisfies (17).

Conversely let $\left(x_{1}^{o}, x_{2}^{o}, u\right) \in \mathbb{R}^{n} \times \mathbb{R}^{n} \times \mathscr{U}$ satisfy (16) and (17). Therefore, $\mathrm{F}\left(\cdot, C, A, x_{o}\right)$ vanishes identically and one has $\mathrm{F}(s, C, A, B) U(s)=C A^{2 n-1} B U(s)=0$ for every $s \in \mathscr{H}_{\theta_{u}} \cap \mathscr{H}_{\boldsymbol{\lambda}^{\star}}$. We conclude that for every $\theta>\boldsymbol{\lambda}^{\star}+\theta_{u}$,

$$
\mathrm{F}(s, C, A, B) U(s)+\mathrm{F}\left(s, C, A, x_{o}\right)=0 .
$$

Hence $\left(x_{1}^{o}, x_{2}^{o}, u\right) \in \mathscr{Z}_{\text {ind }}\left(S_{1}, S_{2}\right)$.

Remark 10. The results of Theorem 9 go through unaltered for multiple output systems using the same approach. This is no longer the case for the results of the next subsection, in particular Theorem 16.

B. Characterization of the indistinguishability zone: the case $k_{0}<2 n-1$.

In what follows, $J(S)$ is the non-empty set defined by

$$
J(S)=\left\{j \in\{1,2, \ldots, l\}: C A^{k_{0}} \mathbf{B}^{j} \neq 0\right\} .
$$

This subsection aims to characterize the indistinguishability zone of $S_{1}$ and $S_{2}$ by solving (11) in the case where $k_{0}<2 n-1$. Note that, given $x_{o},(11)$ is a linear equation for $U$. It can be solved by adding to a particular solution $\mathbf{U}^{\text {par }}$ of this equation, the general solution $\mathbf{U}^{\text {hom }}$ of the homogeneous equation

$$
\mathrm{F}(s, C, A, B) \mathbf{U}^{h o m}(s)=0 \quad \forall s \in \mathscr{H}_{\boldsymbol{\theta}_{1}^{\star}}
$$

where

$$
\boldsymbol{\theta}_{1}^{\star}=\max \left(\boldsymbol{\lambda}^{\star}, \boldsymbol{\delta}_{F}\right)
$$

with $\boldsymbol{\delta}_{F}=\max _{1 \leq j \leq l}\left\{\Re(s): \mathrm{F}\left(s, C, A, \mathbf{B}^{j}\right)=0\right\}$.

We turn to solve first the homogeneous equation. As it is assumed that the control inputs belong to $\mathscr{C}_{\mathscr{P}}^{\text {ord }}\left(\mathbb{R}_{+}, \mathbb{R}^{l}\right), \mathbf{U}^{\text {hom }}$ has to be determined so that $\mathbf{u}^{\text {hom }} \in \mathscr{C}_{\mathscr{P}}^{\text {ord }}\left(\mathbb{R}_{+}, \mathbb{R}^{l}\right)$. The form of $\mathbf{u}^{\text {hom }}$ is given by Theorem 12. The proof of this result uses Lemma 11 and the expression (22) of $\operatorname{Ker}(\mathrm{F}(s, C, A, B))$ when $s \in \mathscr{H}_{\boldsymbol{\theta}_{1}^{\star}}$.

As $\mathrm{F}(s, C, A, B)$ is a non-zero row vector for every $s \in \mathscr{H}_{\delta_{F}}$, it is clear that

$$
\operatorname{dim}(\operatorname{Ker}(\mathrm{F}(s, C, A, B)))=l-1
$$


and that for any $j_{0} \in J(S)$,

$$
\operatorname{Ker}(\mathrm{F}(s, C, A, B))=\operatorname{span}\left\{\begin{array}{l}
\Psi_{1}^{j_{0}}(s), \ldots, \Psi_{j_{0}-1}^{j_{0}}(s), \\
\Psi_{j_{0}+1}^{j_{0}}(s), \ldots, \Psi_{l}^{j_{0}}(s)
\end{array}\right\}
$$

where the components $\Psi_{i k}^{j_{0}}(s), k=1,2, \ldots, l$ of the vectors $\Psi_{i}^{j_{0}}(s) \in \mathbb{R}^{l}\left(i \neq j_{0}\right)$ are defined as follows:

$$
\Psi_{i k}^{j_{0}}(s)= \begin{cases}1 & \text { if } k=i, \\ -\frac{\mathrm{F}\left(s, C, A, \mathbf{B}^{i}\right)}{\mathrm{F}\left(s, C, A, \mathbf{B}^{j_{0}}\right)} & \text { if } k=j_{0}, \\ 0 & \text { if } k \neq j_{0} \text { and } k \neq i .\end{cases}
$$

Notice that it follows from Lemma (11)-(ii) and the definition of $\boldsymbol{\delta}_{F}$ that for any $j_{0} \in J(S), \mathrm{F}\left(s, C, A, \mathbf{B}^{j_{0}}\right) \neq 0$ for every $s \in$ $\mathscr{H}_{\delta_{F}}$. Consequently, the component functions $\Psi_{i k}^{j_{0}}$ are well defined on $\mathscr{H}_{\delta_{F}}$.

Lemma 11. Let $q \in\{1,2, \ldots, l\}$.

(i) If $q \notin J(S)$ then $\operatorname{deg}\left[\mathrm{F}\left(\cdot, C, A, \mathbf{B}^{q}\right)\right]<2 n-1-k_{0}$.

(ii) If $q \in J(S)$ then $\operatorname{deg}\left[\mathrm{F}\left(\cdot, C, A, \mathbf{B}^{q}\right)\right]=2 n-1-k_{0}$.

Proof. Since $C A^{r} B=0_{1 \times l}$ for every $r=0,1, \ldots, k_{0}-1$, it follows from Lemma 6 that $\mathrm{D}_{2 n-1-r}(B)=0_{1 \times l}$ for every $r=$ $0,1, \ldots, k_{0}-1$. Consequently, $\mathrm{D}_{2 n-1-r}\left(\mathbf{B}^{q}\right)=0$ for every $r=$ $0,1, \ldots, k_{0}-1$ and hence

$$
\begin{aligned}
\mathrm{F}\left(s, C, A, \mathbf{B}^{q}\right)= & C A^{k_{0}} \mathbf{B}^{q} s^{2 n-1-k_{0}} \\
& +\sum_{r=k_{0}+1}^{2 n-1} \mathrm{D}_{2 n-1-r}\left(\mathbf{B}^{q}\right) s^{2 n-1-r} .
\end{aligned}
$$

The rest of the proof follows from the fact that $C A^{k_{0}} \mathbf{B}^{q}=0$ if $q \notin J(S)$ and $C A^{k_{0}} \mathbf{B}^{q} \neq 0$ if not.

Theorem 12. Suppose $k_{0}<2 n-1$ and let $j_{0} \in J(S)$. Then the following statements are equivalent:

(i) $\mathbf{u}^{\text {hom }} \in \mathscr{U}$ and the Laplace transform $\mathbf{U}^{\text {hom }}$ of $\mathbf{u}^{\text {hom }}$ solves (20).

(ii) There exist $l-1$ functions $\omega_{k} \in \mathscr{C}_{\mathscr{P}}^{\text {ord }}\left(\mathbb{R}_{+}, \mathbb{R}\right), k \in$ $\{1,2, \ldots, l\} \backslash\left\{j_{0}\right\}$ such that

$$
\mathbf{u}_{k}^{\text {hom }}=\omega_{k}, \quad \forall k \in\{1,2, \ldots, l\} \backslash\left\{j_{0}\right\}
$$

and

$$
\mathbf{u}_{j_{0}}^{\text {hom }}=-\sum_{j_{0} \neq k=1}^{l} \omega_{k} * \mathcal{L}^{-1}\left[\frac{\mathrm{F}\left(\cdot, C, A, \mathbf{B}^{k}\right)}{\mathrm{F}\left(\cdot, C, A, \mathbf{B}^{j_{0}}\right)}\right]
$$

where $\mathcal{L}^{-1}$ denotes the inverse Laplace transform operator and for every $k \in\{1,2, \ldots, l\}, \mathbf{u}_{k}^{\text {hom }}$ stands for the $k^{\text {th }}$ component of $\mathbf{u}^{\text {hom }}$.

Proof. (i) $\Longrightarrow$ (ii) Suppose $\mathbf{U}^{\text {hom }}$ solves (20). Then it follows from (22) that there exists $l-1$ functions $\Omega_{k}, k \in\{1,2, \ldots, l\} \backslash\left\{j_{0}\right\}$ such that for every $s \in \mathscr{H}_{\boldsymbol{\theta}_{1}^{\star}}$,

$$
\mathbf{U}^{\text {hom }}(s)=\sum_{j_{0} \neq k=1}^{l} \Omega_{k}(s) \Psi_{k}^{j_{0}}(s) .
$$

Thus it follows from (26) and (23) that the components $\mathbf{U}_{k}^{\text {hom }}$ of $\mathbf{U}^{\text {hom }}$ are such that

$$
\mathbf{U}_{k}^{h o m}(s)=\Omega_{k}(s) \quad \forall k \in\{1,2, \ldots, l\} \backslash\left\{j_{0}\right\}
$$

and

$$
\mathbf{U}_{j_{0}}^{h o m}(s)=-\sum_{j_{0} \neq k=1}^{l} \Omega_{k}(s) \frac{\mathrm{F}\left(s, C, A, \mathbf{B}^{k}\right)}{\mathrm{F}\left(s, C, A, \mathbf{B}^{j_{0}}\right)} .
$$

Relation (27) implies that $\Omega_{k}$ is the Laplace transform of $\mathbf{u}_{k}^{\text {hom }}$ and we can define $\omega_{k}=\mathbf{u}_{k}^{\text {hom }} \in \mathscr{C}_{\mathscr{P}}^{\text {ord }}\left(\mathbb{R}_{+}, \mathbb{R}\right)$. Furthermore, since $\omega_{k} \in \mathscr{C}_{\mathscr{P}}^{\text {ord }}\left(\mathbb{R}_{+}, \mathbb{R}\right)$ and since $\frac{\mathrm{F}\left(s, C, A, \mathbf{B}^{k}\right)}{\mathrm{F}\left(s, C, A, \mathbf{B}^{j_{0}}\right)}$ is proper or strictly proper (see Lemma 11),

$$
\omega_{k} * \mathcal{L}^{-1}\left[\frac{\mathrm{F}\left(\cdot, C, A, \mathbf{B}^{k}\right)}{\mathrm{F}\left(\cdot, C, A, \mathbf{B}^{j_{0}}\right)}\right] \in \mathscr{C}_{\mathscr{P}}^{\text {ord }}\left(\mathbb{R}_{+}, \mathbb{R}\right)
$$

and it follows from the property of the Laplace transform that

$$
\omega_{k} * \mathcal{L}^{-1}\left[\frac{\mathrm{F}\left(\cdot, C, A, \mathbf{B}^{k}\right)}{\mathrm{F}\left(\cdot, C, A, \mathbf{B}^{j_{0}}\right)}\right]=\mathcal{L}^{-1}\left[\Omega_{k}(\cdot) \frac{\mathrm{F}\left(\cdot, C, A, \mathbf{B}^{k}\right)}{\mathrm{F}\left(\cdot, C, A, \mathbf{B}^{j_{0}}\right)}\right]_{(29)} .
$$

Hence using Lerch's Theorem and the linearity of the operator $\mathcal{L}^{-1}$, it is straightforward to verify that (28) and (29) imply (25).

(ii) $\Longrightarrow$ (i) Suppose there exist $l-1$ functions $\omega_{k} \in \mathscr{C}_{\mathscr{P}}^{\text {ord }}\left(\mathbb{R}_{+}, \mathbb{R}\right)$, $k \in\{1,2, \ldots, l\} \backslash\left\{j_{0}\right\}$ for which $\mathbf{u}^{\text {hom }}$ satisfies (24) and (25). Then denoting by $\Omega_{k}$ the Laplace transform of $\omega_{k}$ and applying the Laplace transform to (24) and (25), one obtains respectively (27) and (28). Consequently, we conclude from (22) and (23) that $\mathbf{U}^{\text {hom }}$ solves (20).

We now turn to the task of determining a particular solution of (11). Lemmas 13 and 14 are technical results needed for the proof of Theorem 15 .

Lemma 13. Let $j \in J(S)$ and let $\Phi_{j}\left(\cdot, A, B, C, x_{o}\right)$ be the rational function defined by

$$
\Phi_{j}\left(s, A, B, C, x_{o}\right)=\frac{\mathrm{F}\left(s, C, A, x_{o}\right)}{\mathrm{F}\left(s, C, A, \mathbf{B}^{j}\right)} .
$$

If $k_{0}<2 n-1$ then $\Phi_{j}\left(\cdot, A, B, C, x_{o}\right)$ is strictly proper if and only if

$$
x_{o} \in \operatorname{Ker}\left(\mathcal{O}^{\left[k_{0}\right]}\right) .
$$

Proof. Suppose $k_{0}<2 n-1$ and let $j \in J(S)$. Since it follows from Lemma 11 that $\operatorname{deg}\left[\mathrm{F}\left(\cdot, C, A, \mathbf{B}^{j}\right)\right]=2 n-1-k_{0}$, $\Phi_{j}\left(\cdot, A, B, C, x_{o}\right)$ is strictly proper if and only if

$$
\operatorname{deg}\left[\mathrm{F}\left(\cdot, C, A, x_{o}\right)\right] \leq 2 n-2-k_{0} .
$$

Moreover, since (32) is equivalent to $\mathrm{D}_{2 n-1-r}\left(x_{o}\right)=0$ for every $r=0,1, \ldots, k_{0}$, one can conclude with Lemma 6 that $\Phi_{j}\left(\cdot, A, B, C, x_{o}\right)$ is strictly proper if and only if $C A^{r} x_{o}=0$, $\forall r=0,1, \ldots, k_{0}$. This concludes the proof.

Lemma 14. If $k_{0}<2 n-1$ and $\left(x_{1}^{o}, x_{2}^{o}, u\right)$ satisfies (11) then the vector $x_{o}=\left[\begin{array}{ll}\left(x_{1}^{o}\right)^{T} & \left(x_{2}^{o}\right)^{T}\end{array}\right]^{T}$ satisfies (31).

Proof. Suppose $k_{0}<2 n-1$. Let $\left(x_{1}^{o}, x_{2}^{o}, u\right)$ satisfy (11) and let $j \in J(S)$. Then $\mathrm{F}\left(\cdot, C, A, \mathbf{B}^{j}\right)$ does not vanish identically and it follows from (11) that

$$
U_{j}(s)=-\frac{\mathrm{F}\left(s, C, A, x_{o}\right)}{\mathrm{F}\left(s, C, A, \mathbf{B}^{j}\right)}-\sum_{j \neq k=1}^{l} U_{k}(s) \frac{\mathrm{F}\left(s, C, A, \mathbf{B}^{k}\right)}{\mathrm{F}\left(s, C, A, \mathbf{B}^{j}\right)}
$$

By a similar reasoning as in the proof of Theorem 12, one can easily show from Lemma 11, and the definition of the Laplace transform of the convolution of functions that

$$
\mathcal{L}^{-1}\left[U_{k}(\cdot) \frac{\mathrm{F}\left(\cdot, C, A, \mathbf{B}^{k}\right)}{\mathrm{F}\left(\cdot, C, A, \mathbf{B}^{j}\right)}\right]=u_{k} * \mathcal{L}^{-1}\left[\frac{\mathrm{F}\left(\cdot, C, A, \mathbf{B}^{k}\right)}{\mathrm{F}\left(\cdot, C, A, \mathbf{B}^{j}\right)}\right]
$$

belongs to $\mathscr{C}_{\mathscr{P}}^{\text {ord }}\left(\mathbb{R}_{+}, \mathbb{R}\right)$. Consequently, since $u_{j}$ is continuous, (33) is true only if $\frac{\mathrm{F}\left(\cdot, C, A, x_{o}\right)}{\mathrm{F}\left(\cdot, C, A, \mathbf{B}^{j}\right)}=\Phi_{j}\left(\cdot, A, B, C, x_{o}\right)$ is strictly proper. Hence from Lemma $13, x_{o}$ satisfies (31).

Theorem 15. Assume that $k_{0}<2 n-1$. Let $x_{1}^{o}$ and $x_{2}^{o}$ be two vectors of $\mathbb{R}^{n}$ such that $x_{o}=\left[\begin{array}{ll}\left(x_{1}^{o}\right)^{T} & \left(x_{2}^{o}\right)^{T}\end{array}\right]^{T}$ satisfies (31) and 
let $\mathbf{u}^{\text {par }}$ be the control input with components $\mathbf{u}_{j}^{\text {par }}, j=1,2, \ldots, l$ defined by:

$$
\mathbf{u}_{j}^{\text {par }}= \begin{cases}0 & \text { if } j \notin J(S), \\ -\frac{\mathcal{L}^{-1}\left[\Phi_{j}\left(\cdot, A, B, C, x_{o}\right)\right]}{\#(J(S))} & \text { if not, }\end{cases}
$$

where \# $(J(S))$ denotes the cardinality of $J(S)$. Then $\mathbf{u}^{\text {par }} \in \mathscr{U}$ and moreover, the Laplace transform $\mathbf{U}^{\text {par }}$ of $\mathbf{u}^{\text {par }}$ and $\left(x_{1}^{o}, x_{2}^{o}\right)$ satisfy (11) for $s \in \mathscr{H}_{\theta_{2}^{\star}}$ where $\boldsymbol{\theta}_{2}^{\star}=\max \left(\theta_{\mathbf{u}^{\text {par }}}, \boldsymbol{\lambda}^{\star}, \boldsymbol{\delta}_{F}\right)$.

Proof. Since $\Phi_{j}\left(\cdot, A, B, C, x_{o}\right)$ is strictly proper (see Lemma 13) then each component $\mathbf{u}_{j}^{\text {par }}$ of $\mathbf{u}^{\text {par }}$ is a smooth function with an exponential order at infinity. Consequently, the control input $\mathbf{u}^{\text {par }}$ satisfies Assumption 4. It follows from (30) and (34) that for every $j \in J(S)$ and for every $s \in \mathscr{H}_{\boldsymbol{\theta}_{2}^{\star}}$,

$$
\mathrm{F}\left(s, C, A, \mathbf{B}^{j}\right) \mathbf{U}_{j}^{\text {par }}(s)+\frac{1}{\#(J(S))} \mathrm{F}\left(s, C, A, x_{o}\right)=0 .
$$

Moreover, since it follows from (10) and (34) that

$$
\mathrm{F}(s, C, A, B) \mathbf{U}^{\text {par }}(s)=\sum_{j \in J(S)} \mathrm{F}\left(s, C, A, \mathbf{B}^{j}\right) \mathbf{U}_{j}^{\text {par }}(s),
$$

(35) implies that for every $s \in \mathscr{H}_{\boldsymbol{\theta}_{2}^{\star}}$

$$
\mathrm{F}(s, C, A, B) \mathbf{U}^{\text {par }}(s)+\mathrm{F}\left(s, C, A, x_{0}\right)=0 .
$$

This concludes the proof.

The indistinguishability zone of $S_{1}$ and $S_{2}$ can be characterized as follows.

Theorem 16. Suppose $k_{0}<2 n-1$. Then $\left(x_{1}^{o}, x_{2}^{o}, u\right) \in$ $\mathscr{Z}_{\text {ind }}\left(S_{1}, S_{2}\right)$ if and only if $x_{o}=\left[\begin{array}{ll}\left(x_{1}^{o}\right)^{T} & \left(x_{2}^{o}\right)^{T}\end{array}\right]^{T}$ satisfies (31) and $u=\mathbf{u}^{\text {hom }}+\mathbf{u}^{\text {par }}$ where $\mathbf{u}^{\text {hom }}$ and $\mathbf{u}^{\text {par }}$ are defined in Lemma 12 and Lemma 15, respectively.

Proof. The proof is an immediate consequence of Lemma 5, Lemma 14, Theorem 12 and Theorem 15.

Notice that for SISO systems $S_{1}$ and $S_{2}(l=1)$, it follows from (21) that $\operatorname{dim}(\operatorname{Ker}(\mathrm{F}(s, C, A, B)))=0, \forall s \in \mathscr{H}_{\boldsymbol{\delta}_{F}}$. Consequently $\mathbf{u}^{\text {hom }}=0_{\mathscr{U}}$. Moreover, as $B=\mathbf{B}^{1}$ and $J(S)=\{1\}$, it follows from Theorem 16 that the control input $u$ that generates identical outputs $y_{1}$ and $y_{2}$ is such that

$$
u=\mathbf{u}^{\text {par }}=\mathbf{u}_{1}^{\text {par }}=-\mathcal{L}^{-1}\left[\Phi_{1}\left(\cdot, A, B, C, x_{o}\right)\right] .
$$

This is the situation of Example 2, when $\tilde{n}=1, M_{1} \neq M_{2}$ and $K_{1} \neq K_{2}$.

\section{CONDITION FOR STRICT DISTINGUISHABILITY OF SINGLE OUTPUT SYSTEMS}

The aim of this section is to derive, from the characterization of the indistinguishability zone, simple conditions allowing one to verify whether or not a couple of SISO or MISO dynamical LTI systems are strictly distinguishable. It was recently shown in [4], [7] that strict distinguishability is equivalent to an appropriate rank condition on a matrix valued function on the complex plane. Theorem 20 gives a necessary and sufficient condition for strict distinguishability for SISO systems. This condition requires checking the observability of $S$ and the values of its Markov parameters. It is therefore much simpler than the condition of [7] which requires checking the rank condition of a matrix valued function. Theorem 22 confirms the result of Theorem 4.5 of [4] which states that two MISO systems $S_{1}$ and $S_{2}$ cannot be strictly distinguishable.

Definition 17 recalls the notion of strict distinguishability.
Definition 17. The systems $S_{1}$ and $S_{2}$ are strictly distinguishable on a time interval $[0 ; \tau]$ if for any non-zero triplet $\left(x_{1}^{o}, x_{2}^{o}, u\right) \in$ $\mathbb{R}^{n} \times \mathbb{R}^{n} \times L^{1}\left([0 ; \boldsymbol{\tau}], \mathbb{R}^{l}\right)$ the outputs $y_{1}\left(\cdot, x_{1}^{o}, u\right)$ and $y_{2}\left(\cdot, x_{2}^{o}, u\right)$ are not identical on $[0 ; \boldsymbol{\tau}]$.

Lemmas 18 and 19 are technical results needed for the proof of Theorems 20 and 22. In Lemma 18 it is shown that when $\mathscr{U}=L^{1}\left([0 ; \tau], \mathbb{R}^{l}\right) \supset \mathscr{C}_{\mathscr{P}}^{\text {ord }}\left(\mathbb{R}_{+}, \mathbb{R}^{l}\right)$, the statements in Theorem 9-(ii) still hold.

Lemma 18. Suppose $k_{0}=2 n-1$ and let $\left(x_{1}^{o}, x_{2}^{o}, u\right) \in \mathbb{R}^{n} \times \mathbb{R}^{n} \times$ $L^{1}\left([0 ; \tau], \mathbb{R}^{l}\right)$. Then the following statements are equivalent:

(i) $y_{1}\left(\cdot, x_{1}^{o}, u\right)$ and $y_{2}\left(\cdot, x_{2}^{o}, u\right)$ are identical on $[0 ; \boldsymbol{\tau}]$.

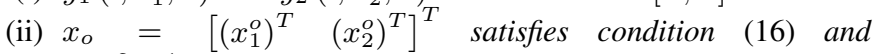
$C A^{2 n-1} B u(t)=0$ for every $t \in[0 ; \tau]$.

Proof. cf. Appendix B.

Lemma 19. If $k_{0} \neq 2 n-1$ then $\mathscr{Z}_{\text {ind }}\left(S_{1}, S_{2}\right) \neq\left\{\left(0_{n}, 0_{n}, 0_{\mathscr{U}}\right)\right\}$.

Proof.

- Suppose $k_{0}<2 n-1$ and let $x_{o}=\left[\begin{array}{ll}\left(x_{1}^{o}\right)^{T} & \left(x_{2}^{o}\right)^{T}\end{array}\right]^{T} \in$ $\operatorname{Ker}\left(\mathcal{O}^{\left[k_{0}\right]}\right)$ be any non zero vector. Notice that the existence of a such vector follows from the fact that $\mathcal{O}^{\left[k_{0}\right]} \in \mathbb{R}^{\left(k_{0}+1\right) \times 2 n}$ is not of full column rank. For this vector, consider the control input $u=\mathbf{u}^{p a r}$ defined by (34). Therefore, $\left(x_{1}^{o}, x_{2}^{o}, u\right)$ is a nonzero triplet and it follows from Theorem 15 that $\left(x_{1}^{o}, x_{2}^{o}, u\right) \in$ $\mathscr{Z}_{\text {ind }}\left(S_{1}, S_{2}\right)$.

- Suppose $k_{0}=2 n$. Let $u \in \mathscr{U}$ be any non-zero control input and

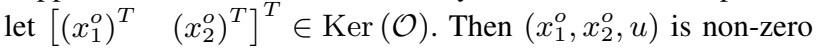
and from Theorem 9, one has $\left(x_{1}^{o}, x_{2}^{o}, u\right) \in \mathscr{Z}_{\text {ind }}\left(S_{1}, S_{2}\right)$.

Theorem 20. Let $S_{1}$ and $S_{2}$ be SISO systems. Then $S_{1}$ and $S_{2}$ are strictly distinguishable on $[0 ; \tau]$ if and only if $k_{0}=2 n-1$ and $\operatorname{Rank}(\mathcal{O})=2 n$.

Proof. First, suppose $k_{0} \neq 2 n-1$ and let $\left(x_{1}^{o}, x_{2}^{o}, u\right) \in$ $\mathscr{Z}_{\text {ind }}\left(S_{1}, S_{2}\right)$ such that $\left(x_{1}^{o}, x_{2}^{o}, u\right) \neq\left(0_{n}, 0_{n}, 0_{\mathscr{U}}\right)$. The existence of a such triplet follows from Lemma 19. Then $u$ is Lebesgue integrable on $[0 ; \boldsymbol{\tau}]$ (because $u$ is continuous on $\mathbb{R}_{+}$) and the outputs $y_{1}\left(\cdot, x_{1}^{o}, u\right)$ and $y_{2}\left(\cdot, x_{2}^{o}, u\right)$ are identical on $[0 ; \boldsymbol{\tau}]$. Consequently, when $k_{0} \neq 2 n-1, S_{1}$ and $S_{2}$ are not strictly distinguishable on $[0 ; \boldsymbol{\tau}]$. Therefore to conclude the proof, we will show that if $k_{0}=2 n-1$ then $S_{1}$ and $S_{2}$ are strictly distinguishable if and only if $\operatorname{Rank}(\mathcal{O})=2 n$.

Now suppose $k_{0}=2 n-1$. It follows from definition 17 that $S_{1}$ and $S_{2}$ are strictly distinguishable if and only if

$$
\left.\begin{array}{l}
\left(x_{1}^{o}, x_{2}^{o}, u\right) \in \mathscr{V}, \\
y_{1}\left(t, x_{1}^{o}, u\right)=y_{2}\left(t, x_{2}^{o}, u\right), \\
\forall t \in[0 ; \boldsymbol{\tau}],
\end{array}\right\} \Longrightarrow\left\{\begin{array}{l}
x_{1}^{o}=x_{2}^{o}=0_{n} \\
u(t)=0 \\
\forall t \in[0 ; \boldsymbol{\tau}]
\end{array}\right.
$$

where $\mathscr{V}=\mathbb{R}^{n} \times \mathbb{R}^{n} \times L^{1}([0 ; \tau], \mathbb{R})$. Moreover, since it follows from Lemma 18 that $y_{1}\left(\cdot, x_{1}^{o}, u\right)$ and $y_{2}\left(\cdot, x_{2}^{o}, u\right)$ are identical on $[0 ; \boldsymbol{\tau}]$ if and only if for every $t \in[0 ; \boldsymbol{\tau}], C A^{2 n-1} B u(t)=0$ and $\left[\begin{array}{ll}\left(x_{1}^{o}\right)^{T} & \left(x_{2}^{o}\right)^{T}\end{array}\right]^{T} \in \operatorname{Ker}(\mathcal{O})$, then $S_{1}$ and $S_{2}$ are strictly distinguishable on $[0 ; \tau]$ if and only if

$$
\left.\begin{array}{l}
{\left[\left(x_{1}^{o}\right)^{T}\left(x_{2}^{o}\right)^{T}\right]^{T} \in \operatorname{Ker}(\mathcal{O}),} \\
C A^{2 n-1} B u(t)=0, \forall t \in[0 ; \boldsymbol{\tau}],
\end{array}\right\} \Longrightarrow\left\{\begin{array}{l}
x_{1}^{o}=x_{2}^{o}=0_{n}, \\
u(t)=0, \forall t \in[0 ; \boldsymbol{\tau}] .
\end{array}\right.
$$

Consequently, as $C A^{2 n-1} B \neq 0$ (see definition (15) of $k_{0}$ ), we conclude that $S_{1}$ and $S_{2}$ are strictly distinguishable if and only if $\operatorname{Ker}(\mathcal{O})=\left\{0_{2 n}\right\}$. This concludes the proof. 
So, the only theoretical situation where a couple of SISO systems can be strictly distinguishable is the case where their augmented system $S$ is observable and among its first $2 n-1$ Markov parameters, only the $(2 n-1)^{t h}$ one is non-zero.

Example 21. Consider the two harmonic systems of Example 7. If $M_{1} \neq M_{2}$ or $K_{1}=K_{2}$ one has $k_{0} \neq 3=2 n-1$. However, when $M_{1}=M_{2}$ and $K_{1} \neq K_{2}$ one has $k_{0}=3$ and $\operatorname{Rank}(\mathcal{O})=4=2 n$.

Theorem 22. Let $S_{1}$ and $S_{2}$ be MISO systems. Then $S_{1}$ and $S_{2}$ are not strictly distinguishable on $[0 ; \boldsymbol{\tau}]$.

Proof. If $k_{0} \neq 2 n-1$, then using the same reasoning as that of the first part of the proof of Theorem 22, it is straightforward to verify that $S_{1}$ and $S_{2}$ are not strictly distinguishable on $[0 ; \tau]$.

Now suppose $k_{0}=2 n-1$ and let $v^{\star} \in \operatorname{Ker}\left(C A^{2 n-1} B\right)$ such that $v^{\star} \neq 0_{l}$. The existence of a such vector $v^{\star}$ follows from the fact that the matrix $C A^{2 n-1} B \in \mathbb{R}^{1 \times l}$ is not of full column rank. Let $u$ be the input defined by $u(t)=v^{\star}, \quad \forall t \geq 0$ and let $x_{1}^{o} \in \mathbb{R}^{n}$ and $x_{2}^{o} \in \mathbb{R}^{n}$ such that $\left[\left(x_{1}^{o}\right)^{T} \quad\left(x_{2}^{o}\right)^{T}\right]^{T} \in \operatorname{Ker}(\mathcal{O})$. Then $u$ is is Lebesgue integrable on $[0 ; \boldsymbol{\tau}]$ and $\left(x_{1}^{o}, x_{2}^{o}, u\right) \neq\left(0_{n}, 0_{n}, 0 \mathscr{U}\right)$. Moreover, it follows from Theorem 9 that the outputs $y_{1}\left(\cdot, x_{1}^{o}, u\right)$ and $y_{2}\left(\cdot, x_{2}^{o}, u\right)$ are identical on $[0 ; \tau]$.Therefore, $S_{1}$ and $S_{2}$ are not strictly distinguishable on $[0 ; \boldsymbol{\tau}]$.

\section{CONCLUSION}

In this paper, we have proposed a novel approach to the distinguishability of two LTI systems, based on the notion of "indistinguishability zone" of the systems. Its characterization permits us to identify exactly the set of Laplace transformable control inputs that can generate the same output signals for two distinct single output systems. Note that this result allows one in principle to check whether given inputs may lead to indistinguishable outputs for the two systems. We have presented two applications of this new approach. First, we have given a simple necessary and sufficient condition for testing the strict distinguishability of SISO systems. Second, we provided a new and simple proof of the fact that MISO systems are never strictly distinguishable, first proven in [2].

\section{APPENDIX A}

\section{PROOF OF LEMMA 6}

Notice that for every $r_{0} \in\{0,1, \ldots, 2 n-1\}$, (ii) $\Longrightarrow$ (i) because for every $r=0,1, \ldots, r_{0}, \mathrm{D}_{2 n-1-r}(M)$ depends linearly on $C A^{k} M, k=0,1, \ldots, r$. Now we shall prove by recurrence on $r_{0} \in\{0,1, \ldots, 2 n-1\}$ that (i) $\Longrightarrow$ (ii). Let $\mathcal{P}_{r_{0}}$ be the proposition (i) $\Longrightarrow$ (ii) i.e.

$$
\left.\mathcal{P}_{r_{0}}: \begin{array}{c}
\mathrm{D}_{2 n-1-r}(M)=0_{1 \times q}, \\
r=0,1, \ldots, r_{0}
\end{array}\right\} \Longrightarrow\left\{\begin{array}{c}
C A^{r} M=0_{1 \times q}, \\
r=0,1, \ldots, r_{0} .
\end{array}\right.
$$

Since it follows from (14) that $\mathrm{D}_{2 n-1}(M)=C M$,

$$
\mathrm{D}_{2 n-1}(M)=0_{1 \times q} \Longrightarrow C M=0_{1 \times q} .
$$

Hence $\mathcal{P}_{0}$ is true. Now, suppose $r_{0} \in\{1,2, \ldots, 2 n\}$ and that $\mathcal{P}_{r_{0}-1}$ is true. Assume that

$$
\mathrm{D}_{2 n-1-r}(M)=0_{1 \times q} \quad \forall r=0,1, \ldots, r_{0} .
$$

Then $\forall r=0,1, \ldots, r_{0}-1, \mathrm{D}_{2 n-1-r}(M)=0_{1 \times q}$ and since $\mathcal{P}_{r_{0}-1}$ is true,

$$
C A^{r} M=0_{1 \times q} \quad \forall r=0,1, \ldots, r_{0}-1 .
$$

So to conclude the proof, we shall prove that $C A^{r_{0}} M=0_{1 \times q}$. Since $\mathrm{D}_{2 n-1-r_{0}}(M)=0_{1 \times q}$ (see (36)), it follows from (14) that

$$
\sum_{k=0}^{r_{0}-1} \alpha_{2 n-r_{0}+k} C A^{k} M+C A^{r_{0}} M=0_{1 \times q} .
$$

Hence (37) and (38) imply that $C A^{r_{0}} M=0_{1 \times q}$. This concludes the proof.

\section{APPENDIX B}

PROOF OF LEMMA 18

(i) $\Longrightarrow$ (ii) As $k_{0}=2 n-1, C A^{r} B=0$ for every $r=0,1, \ldots, 2 n-1$. Therefore, by successive derivations of the output equation $y\left(t, x_{1}^{o}, u\right)=C x(t)$ and successive substitution of $\dot{x}(t)=A x(t)+B u(t)$ into $y^{(r)}\left(t, x_{o}, u\right)$, one obtains

$$
y^{(r)}\left(t, x_{o}, u\right)=C A^{r} x(t)+M_{r} u(t), \quad r=0,1, \ldots, 2 n,
$$

with $M_{0}=M_{1}=\cdots=M_{2 n-1}=0$ and $M_{2 n}=C A^{2 n-1} B$. Consequently, $(i)$ implies that for every $t \in[0 ; \boldsymbol{\tau}]$,

$$
C A^{r} x(t)=0, r=0,1, \ldots, 2 n-1,
$$

and

$$
C A^{2 n} x(t)+C A^{2 n-1} B u(t)=0 .
$$

Thus, by rewriting (39) for $t=0$, one obtains the condition (16). It follows from the theorem of Cayley-Hamilton that (39) implies $C A^{2 n} x(t)=0$ for every $t \in[0 ; \boldsymbol{\tau}]$. Consequently, (40) becomes $C A^{2 n-1} B u(t)=0$ for every $t \in[0 ; \tau]$.

(ii) $\Longrightarrow$ (i) Let $t \in[0 ; \boldsymbol{\tau}]$. Condition (16) implies that $C \mathrm{e}^{t A} x_{o}=$ 0 . Moreover, as $C A^{2 n-1} B u(t)=0$ and as $C A^{r} B=0$ for every $r=0,1, \ldots, 2 n-2$, we conclude from the Cayley-Hamilton theorem that $C \mathrm{e}^{t A} B u(t)=0$. Consequently, for every $t \in[0 ; \boldsymbol{\tau}]$,

$$
y\left(t, x_{1}^{o}, u\right)=C \mathrm{e}^{t A} x_{o}+C \int_{0}^{t} \mathrm{e}^{(t-\tau) A} B u(\tau) \mathrm{d} \tau=0 .
$$

\section{REFERENCES}

[1] M. S. Grewal and K. Glover, "Identifiability of linear and nonlinear dynamical system," IEEE Transactions on Automatic Control, vol. 6 , pp. 833-837, 1976.

[2] M. Grewal, G. Bekey, and H. Payne, "Parameter identifiability of dynamical systems," in Proceedings of the 1974 IEEE Conference on Decision and Control including the 13th Symposium on Adaptative Processes, vol. 13, no. 1, Phoenix, AZ, USA, 20-22 November 1974, pp. $446-448$.

[3] M. Babaali and G. Pappas, "Observability of switched linear systems in continuous time," Hybrid Systems: Computation and Control (R. Alur and G. Pappas, eds.). Springers, pp. 48-63, 2004.

[4] H. Lou and P. Si, "The distinguishability of linear control systems," Nonlinear Analysis : Hybrid Systems, vol. 1, pp. 21-38, 2009.

[5] R. Vidal, A. Chiuso, S. Soatto, and S. Sastry, "Observability and identifiability of jump linear systems," in Proceedings of the 6th International Workshop on Hybrid Systems: Computation and Control, HSCC 2003, Springer, Ed., vol. 2623, Prague, Czech Republic, April 3-5 2003, pp. 526-539.

[6] P. Rosa and C. Silvestre, "On the distinguishability of discrete linear time-invariant dynamic systems," in Proceedings of the 50th IEEE Conference on Decision and Control and European Control Conference (CDC-ECC), Orlando, FL, USA, December 12-15 2011, pp. 3356-3361.

[7] H. Lou and R. Yang, "Necessary and sufficient conditions for distinguishability of linear control systems," Acta Mathematicae Applicatae Sinica, English Series, vol. 30, no. 2, pp. 473-482, 2014.

[8] K. M. D. Motchon, "Caractérisation de la discernabilité des systèmes dynamiques linéaires et non-linéaires," Ph.D. dissertation, Univerité Lille 1, 59650 Villeneuve d'Ascq Cedex France, December 2015. [Online]. Available: https://www.researchgate.net/profile/Djidula_Motchon

[9] J. L. Schiff, The Laplace Transform: Theory and Applications, Springer, Ed., 1999.

[10] H. Weber and G. Arfken, Essential Mathematical Methods for Physicists, A. Press, Ed., 2003.

[11] F. Blume and C. Edward, Applied Calculus For Scientists And Engineers. Jones and Bartlett Publishers, 2005.

[12] J. Leyva-Ramos, "A method for partial-fraction expansion of transfer matrices," IEEE Transactions On Automatic Control, vol. 36, no. 12, pp. 1472-1475, 1991.

[13] K. Réaud, J.-M. Souriau, C. Vallée, and D. Fortuné, "Méthode de Le Verrier-Souriau et équations différentielles linéaires," Acoustique, ondes, vibrations, vol. IIb, pp. 773-778, 2000. 